\title{
Quantifying Photoinduced Polaronic Distortions in Inorganic Lead Halide Perovskite Nanocrystals
}

\author{
Oliviero Cannelli, Nicola Colonna, Michele Puppin, Thomas C. Rossi, Dominik Kinschel, \\ Ludmila M. D. Leroy, Janina Löffler, James M. Budarz, Anne Marie March, Gilles Doumy, \\ Andre Al Haddad, Ming-Feng Tu, Yoshiaki Kumagai, Donald Walko, Grigory Smolentsev, \\ Franziska Krieg, Simon C. Boehme, Maksym V. Kovalenko, Majed Chergui,* and Giulia F. Mancini*
}

Cite This: J. Am. Chem. Soc. 2021, 143, 9048-9059

Read Online

ACCESS | W Metrics \& More 喟 Article Recommendations

Supporting Information

ABSTRACT: The development of next-generation perovskitebased optoelectronic devices relies critically on the understanding of the interaction between charge carriers and the polar lattice in out-of-equilibrium conditions. While it has become increasingly evident for $\mathrm{CsPbBr}_{3}$ perovskites that the $\mathrm{Pb}-\mathrm{Br}$ framework flexibility plays a key role in their light-activated functionality, the corresponding local structural rearrangement has not yet been unambiguously identified. In this work, we demonstrate that the photoinduced lattice changes in the system are due to a specific polaronic distortion, associated with the activation of a longitudinal optical phonon mode at $18 \mathrm{meV}$ by electron-phonon coupling, and we quantify the associated structural changes with atomic-level
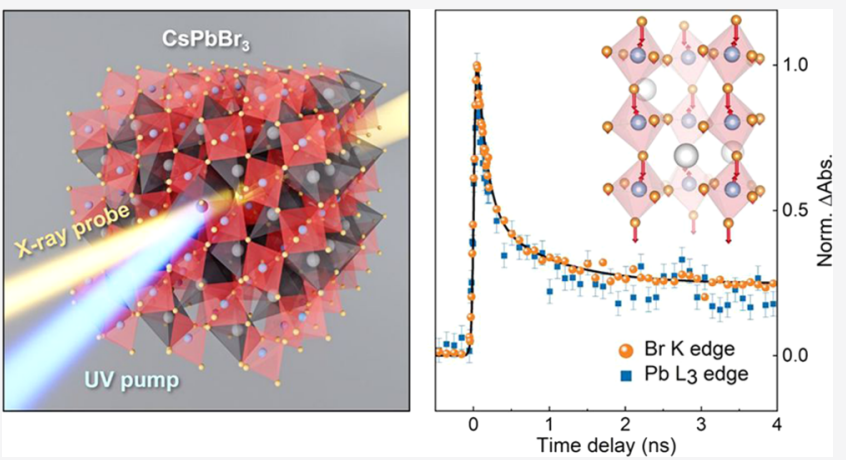
precision. Key to this achievement is the combination of timeresolved and temperature-dependent studies at $\mathrm{Br} \mathrm{K}$ and $\mathrm{Pb} \mathrm{L}_{3} \mathrm{X}$-ray absorption edges with refined ab initio simulations, which fully account for the screened core-hole final state effects on the X-ray absorption spectra. From the temporal kinetics, we show that carrier recombination reversibly unlocks the structural deformation at both $\mathrm{Br}$ and $\mathrm{Pb}$ sites. The comparison with the temperaturedependent XAS results rules out thermal effects as the primary source of distortion of the $\mathrm{Pb}-\mathrm{Br}$ bonding motif during photoexcitation. Our work provides a comprehensive description of the $\mathrm{CsPbBr}_{3}$ perovskites' photophysics, offering novel insights on the light-induced response of the system and its exceptional optoelectronic properties.

\section{INTRODUCTION}

Lead halide perovskites are rapidly emerging as excellent candidates for optoelectronic applications, such as photovoltaics, light-emitting diodes, ${ }^{1}$ lasers, ${ }^{2}$ photodetectors, ${ }^{3}$ polariton devices, ${ }^{4}$ and quantum light sources, ${ }^{5}$ thanks to their outstanding performances and low fabrication costs. ${ }^{6}$ These materials are characterized by facile processing routes, leading to defect-tolerant systems with widely tunable band gaps, high photoluminescence (PL) quantum yields, and narrow emission lines. Their potential stems from their extraordinarily long carrier lifetimes and diffusion lengths, ${ }^{7,8}$ which are in apparent contrast with previously reported low charge mobility ${ }^{9}$ and lattice dynamical disorder. ${ }^{10}$

The $\mathrm{APbX}_{3}$ perovskite structure comprises a $\mathrm{Pb}-\mathrm{X}(\mathrm{X}=\mathrm{Cl}$, $\mathrm{Br}, \mathrm{I})$ inorganic framework made of flexible corner-sharing octahedra, with $\mathrm{Pb}^{2+}$ cations surrounded by six halide anions, characterized by low-frequency phonons and a pronounced anharmonicity. ${ }^{11-15}$ The $\mathrm{A}^{+}$cations, either inorganic $\left(\mathrm{Cs}^{+}\right)$or organic (methylammonium, $\mathrm{MA}^{+}$, or formamidinium, $\mathrm{FA}^{+}$), fill the voids between $\mathrm{PbX}_{6}$ octahedra. Distinct orthorhombic, tetragonal, and cubic phases were identified in these systems, with phase transition temperatures varying with the cation composition. ${ }^{13,16-18}$ In $\mathrm{CsPbBr}_{3}$ nanocrystals (NCs), the phase diagram is characterized by a room-temperature orthorhombic Pnma crystalline group, with a transition to a tetragonal $P 4 \bar{m} b m$ group between 50 and $59{ }^{\circ} \mathrm{C}$, and a higher temperature transition to a cubic $P m \overline{3} m$ group between 108 and $117^{\circ} \mathrm{C}$. ${ }^{13}$

Recent temperature-dependent studies revealed competitive mechanisms underlying the thermal response in lead halide perovskites. Pair distribution function (PDF) analysis from $\mathrm{X}$ ray powder diffraction in organic perovskites at room temperature showed significant internal local distortions of the $\mathrm{PbX}_{6}$ octahedra. ${ }^{19}$ The degree of these distortions was found to increase with the temperature in $\mathrm{MAPbBr}_{3}{ }^{20}$ In $\mathrm{CsPbX}_{3} \mathrm{NCs}$,

Received: March 3, 2021

Published: June 2, 2021 
structural defectiveness was revealed and ascribed to twin boundaries, whose density increases with temperature and leads to an apparent higher-symmetry structure that does, however, not correspond to the $P m \overline{3} m$ cubic phase. ${ }^{12}$ High energy resolution inelastic X-ray scattering and PDF studies on $\mathrm{MAPbI}_{3}$ pointed to the presence of thermally active anharmonic soft modes at $350 \mathrm{~K},{ }^{11}$ and local polar fluctuations among different noncubic structures were confirmed in a low-frequency Raman study on $\mathrm{MAPbBr}_{3}$ and $\mathrm{CsPbBr}_{3}$ perovskites. ${ }^{15}$

The peculiar lattice flexibility of lead halide perovskites also critically underpins their photodynamics. Time-resolved optical studies were conducted to understand key aspects of the early dynamics of the system. ${ }^{21-23}$ Several works suggested the presence of polarons, i.e., charge carriers dressed by lattice distortions, in order to explain the time-resolved optical signals ${ }^{14,24-27}$ and transport properties. ${ }^{28}$ Polaron formation was shown to generally occur through the Fröhlich mechanism, ${ }^{29}$ which corresponds to a Coulomb interaction between the charge carriers and the macroscopic electric field created by the activation of longitudinal optical (LO) phonons. ${ }^{30,31}$

Although the polaron hypothesis was frequently invoked to rationalize experimental observations in both organic and inorganic perovskites, the quantification of the associated local structural rearrangement is still missing. In hybrid organicinorganic lead halide perovskites, local distortions around the $\mathrm{Pb}^{32}$ and $\mathrm{Br}^{33}$ sites were separately reported in time-resolved $\mathrm{X}$ ray absorption spectroscopy (TR-XAS) studies and ascribed to polaron formation, but an unambiguous identification of the associated structural fingerprint was not provided. Ultrafast electron diffraction on a $\mathrm{MAPbI}_{3}$ thin film showed evidence of local rotational disorder of the $\mathrm{PbI}_{6}$ octahedra arising from optical excitation, as a consequence of hot carrier-phonon coupling. ${ }^{34}$ Only recently was the presence of a dynamically expanding polaronic strain structurally identified in an $\mathrm{MAPbBr}_{3}$ single crystal with time-resolved diffusive X-ray scattering. ${ }^{35}$

In all-inorganic lead halide perovskites the picture is still unclear. In a previous TR-XAS study of photoexcited $\mathrm{Cs} \mathrm{PbBr}_{3}$ and $\mathrm{CsPb}(\mathrm{BrCl})_{3} \mathrm{NCs}$ at the $\mathrm{Br} \mathrm{K}$-, $\mathrm{Pb} \mathrm{L}_{3^{-}}$, and $\mathrm{Cs}_{2}$-edges ${ }^{36}$ carried out at a synchrotron, it was suggested that hole polarons form within the time resolution of the experiment ( $\leq 100 \mathrm{ps})$, around $\mathrm{Br}$ centers, with the halide ion turning into a neutral halogen, while electrons would be delocalized in the conduction band (CB). Additionally, the Cs sites did not show any response to photoexcitation. In a more recent time-resolved X-ray diffraction study (TR-XRD) at similar fluences, namely, between 2.5 and $12 \mathrm{~mJ} / \mathrm{cm}^{2}$, the lattice response upon photoexcitation was interpreted in terms of transient amorphization from a crystalline structure. ${ }^{37}$ In both studies, however, the local structural distortion was invoked to rationalize experimental data in a qualitative way, rather than a quantitative one. A recent angle-resolved photoelectron spectroscopy (ARPES) study concluded to the existence of hole polarons based on the increase of the hole effective mass in $\mathrm{CsPbBr}_{3}$ single crystals caused by electron-phonon coupling and it identified a specific LO phonon at $18 \mathrm{meV}$ as the most coupled mode with the charge carriers. ${ }^{38}$

In this work we demonstrate that, in $\mathrm{CsPbBr}_{3} \mathrm{NCs}$, the 18 meV LO phonon mode is underpinning the structural distortion induced upon photoexcitation, and we quantify the polaronic nuclear displacements with atomic precision. Specifically, we conducted a TR-XAS study at the $\mathrm{Br} \mathrm{K}$ and $\mathrm{Pb} \mathrm{L} \mathrm{L}_{3}$ absorption edges and we found that photoexcitation indeed induces polaron formation around $\mathrm{Br}$ centers, which also determines the response of $\mathrm{Pb}$ centers. We performed band structure calculations in which-to our knowledge for the first time in TR-XAS studies-the possible structural distortions are a-priori selected on a physical basis, fully accounting for core-hole final state effects on the XAS spectra. By comparing these accurate simulations to our experimental results, we identify the local $\mathrm{PbX}_{6}$ octahedra bond distortions that contribute to the polaronic photoresponse. Additionally, we clarify the fundamental difference underlying optical and thermal activation in $\mathrm{CsPbBr}_{3}$ NCs: our temperature-dependent XAS experiments result in quantitatively different spectral modifications compared to the optical activation, thereby excluding heat as the primary source of distortion of the $\mathrm{Pb}-\mathrm{Br}$ bonding motif upon photoexcitation.

\section{METHODS}

$\mathrm{Br} \mathrm{K}$-edge and $\mathrm{Pb} \mathrm{L}_{3}$-edge spectra have been recorded in the pre-edge and the XANES (X-ray absorption near edge structure) regions upon light or thermal activation of $\mathrm{CsPBr}_{3}$ NCs. The pre-edge region contains bound-bound core-to-valence transitions and is therefore sensitive to the density of unoccupied valence orbitals. The XANES includes the region just above the ionization limit (i.e., the edge). It is characterized by single and multiple scattering events of the photoelectron, and, hence, it contains information about the bond distances and angles to the nearest-neighbor atoms around the probed site. $^{39,40}$ In its time-resolved implementation, the photoinduced changes of the TR-XAS spectrum reflect transient structural and electronic modifications at the probed sites and in their local environment. $^{41}$

The TR-XAS experiments were conducted at the 7ID-D beamline at the Advanced Photon Source (APS) of the Argonne National Laboratory. ${ }^{42,43}$ A schematic representation of this experiment is shown in Figure 1. The sample consisted of long-chain zwitterion-

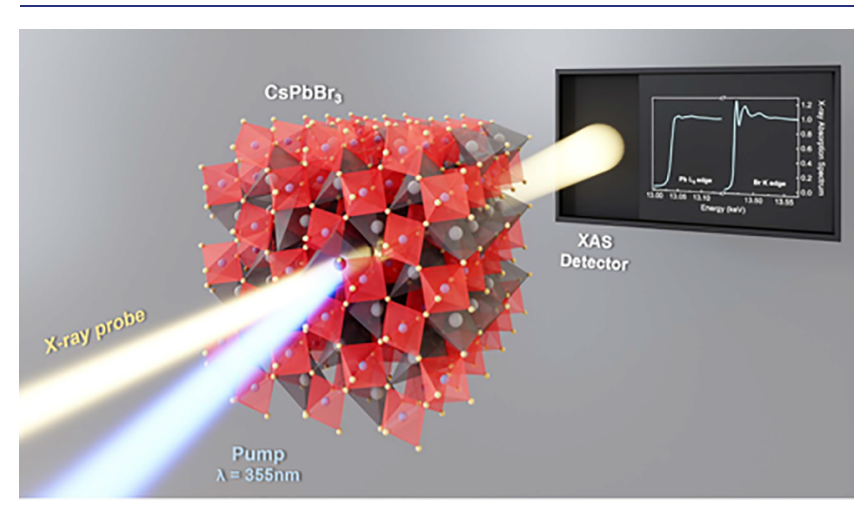

Figure 1. Ultrafast element-selective probing of optically induced polaronic distortions in $\mathrm{CsPbr}_{3}$ perovskite NCs. Schematic layout of the experiment. TR-XAS was conducted on long-chain zwitterioncapped $\mathrm{CsPbrr}_{3} \mathrm{NCs}$ dispersed in a toluene solution with a concentration of $5.8 \mathrm{mg} / \mathrm{mL}$ and flowed through a flat jet. The laser pump $(355 \mathrm{~nm})$ and the $\mathrm{X}$-ray probe at the $\mathrm{Br}$ K-edge (13.450-13.570 $\mathrm{keV})$ and $\mathrm{Pb} \mathrm{L}_{3}$-edge $(13.000-13.140 \mathrm{keV})$ were in an almost collinear geometry. Courtesy of Balázs Ôrley. Please note: Image also appears in the TOC/Graphical Abstract.

capped $\mathrm{CsPbBr}{ }_{3}$ perovskite NCs with cuboidal shape (side length $10 \pm$ $2 \mathrm{~nm}$ ) and high PL quantum yields. ${ }^{44}$ Above band gap photoexcitation was performed using a Duetto laser at a photon energy of $3.49 \mathrm{eV}$ (i.e., $1.1 \mathrm{eV}$ above the direct band gap excitation), a repetition rate of 1.304 $\mathrm{MHz}, 10 \mathrm{ps}$ pulse duration, and with a fluence of $8.8 \mathrm{~mJ} / \mathrm{cm}^{2}$, in the linear response regime. The photoinduced changes in the sample were probed at the Br K-edge (13.45-13.57 keV) and $\mathrm{Pb} \mathrm{L}_{3}$-edge (13.00$13.14 \mathrm{keV}$ ), with $\sim 80$ ps time resolution. 

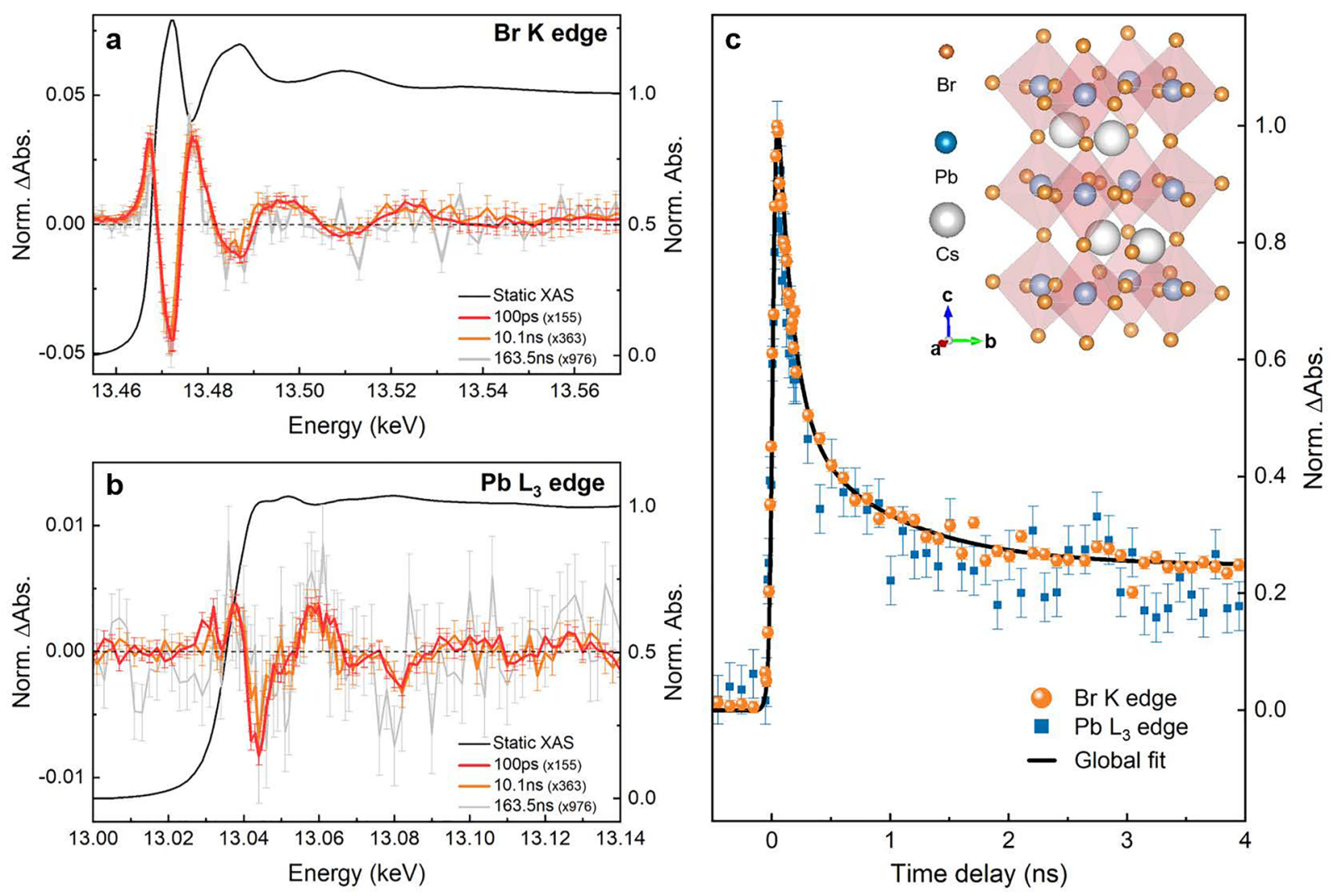

Figure 2. X-ray absorption energy and time traces. (a) Br K-edge and (b) $\mathrm{Pb} \mathrm{L}_{3}$-edge XAS spectra: steady-state (black) and energy transients at 100 ps (red, $\times 155$ ), $10.1 \mathrm{~ns}$ (yellow, $\times 363$ ), and $163.5 \mathrm{~ns}$ (gray, $\times 976$ ) time delays. The error bars correspond to the standard error of the measurements. (c) TR-XAS time traces at the $\mathrm{Br}$ K-edge (13.472 keV, orange), $\mathrm{Pb} \mathrm{L}_{3}$-edge (13.043 keV, light blue), and the exponential fit (black). The error bars were computed as the error propagation of the pumped and unpumped scans, calculated as the square root of the total single-photon counts. Inset: Graphical representation of Pnma orthorhombic $\mathrm{CsPBBr}_{3}{ }^{68} \mathrm{The} \mathrm{Br}, \mathrm{Pb}$, and $\mathrm{Cs}$ atoms are respectively reported as orange, light blue, and gray spheres.

Comparative temperature-dependent $\mathrm{Br} \mathrm{K}$-edge and $\mathrm{Pb} \mathrm{L}_{3}$-edge static XAS was conducted at the SuperXAS beamline of the Swiss Light Source (SLS). The experiments were performed on dry $\mathrm{CsPbBr}_{3} \mathrm{NCs}$ enclosed in a thermostated cell holder. The thermal response of the system was monitored in the temperature range between 25 and 140 ${ }^{\circ} \mathrm{C}$, where effects ascribed to either an increase in the NCs local structural disorder ${ }^{12}$ or the occurrence of orthorhombic-tetragonalcubic phase transitions ${ }^{13,16}$ had previously been reported. Moreover, we acquired for each temperature step XRD patterns at $12.9 \mathrm{keV}$, below both absorption edges, to track longer-range structural changes and to assess the overall quality of the sample.

We performed first-principles calculations using the Quantum ESPRESSO distribution, ${ }^{45,46}$ based on density functional theory (DFT) and plane-wave and pseudopotentials techniques. The Perdew-Burke-Ernzerhof functional ${ }^{47}$ was used to describe electronic exchange-correlation effects. The electron-ion interaction was modeled using ultrasoft pseudopotentials from the PS-library. ${ }^{48}$ The projected density of states ( $\mathrm{p}$-DOS) was computed across the band gap. XANES Br K-edge spectra were simulated with the XSpectra code ${ }^{49,50}$ of Quantum ESPRESSO, explicitly accounting for the screened corehole effect in separate supercell calculations for each nonequivalent $\mathrm{Br}$ atom and calculating the average $\mathrm{Br} \mathrm{K}$-edge spectra. XANES $\mathrm{Pb} \mathrm{L}_{3}$-edge calculations were not carried out due to the limitations in the explicit inclusion of a screened core-hole in describing holes with nonzero orbital momentum, ${ }^{51}$ as in the case of the $\mathrm{Pb} 2 \mathrm{p}_{3 / 2}$ orbital. Details about all experimental methods, the data acquisition scheme, and the computational methods and DOS calculations are described in the Supporting Information (SI).

\section{RESULTS}

TR-XAS. The steady-state $\mathrm{Br} \mathrm{K}$-edge and $\mathrm{Pb} \mathrm{L}_{3}$-edge spectra, normalized to the last data point of the postedge region, are shown in Figure 2a and b (black solid line). Our calculations of the p-DOS show that the top of the valence band (VB) is composed of $\mathrm{Br} 4 \mathrm{p}$ orbitals, with a non-negligible proportion of $\mathrm{Pb} 6$ s orbitals and a minor contribution of $\mathrm{Pb} 6 \mathrm{p}$ orbitals, while the $\mathrm{CB}$ is largely dominated by the $\mathrm{Pb} 6 \mathrm{p}$ orbitals (see the $\mathrm{SI}$ ).

The photoinduced changes are reflected in the transient spectra, defined as the difference of the excited minus unexcited XAS spectra, and shown for UV pump/X-ray probe time delays of $100 \mathrm{ps}$ (red), $10.1 \mathrm{~ns}$ (yellow), and $163.5 \mathrm{~ns}$ (gray). The $\mathrm{Br}$ transient spectra (Figure $2 a$ ) were scaled by the inverse of the absolute area underlying the curves, i.e., $\times 155$ (100 ps), $\times 363$ (10.1 ns), and $\times 976$ (163.5 ns). The same scaling factors were used for the $\mathrm{Pb}$ transients (Figure $2 \mathrm{~b}$, details in the SI). Notably, even though the amplitude of the TR-XAS decays over time, the profiles of both $\mathrm{Br}$ and $\mathrm{Pb}$ transient spectra remain unchanged.

The $\mathrm{Br}$ K-edge transients show prominent peaks at the preedge $(13.4675 \mathrm{keV})$, main-edge $(13.472 \mathrm{keV})$, and postedge $(13.4765 \mathrm{keV})$ regions. The first feature was ascribed to the opening of a new $1 s-4 p$ channel following the creation of holes in the VB upon photoexcitation. ${ }^{36}$ Such a scenario also implies a blue shift of the edge, and, indeed, the second and third features could partially be reproduced in the difference spectrum of the blue-shifted ground-state spectrum minus the unshifted one. However, this qualitative approach does not account for all the modulations that show up in the above-edge region, which 

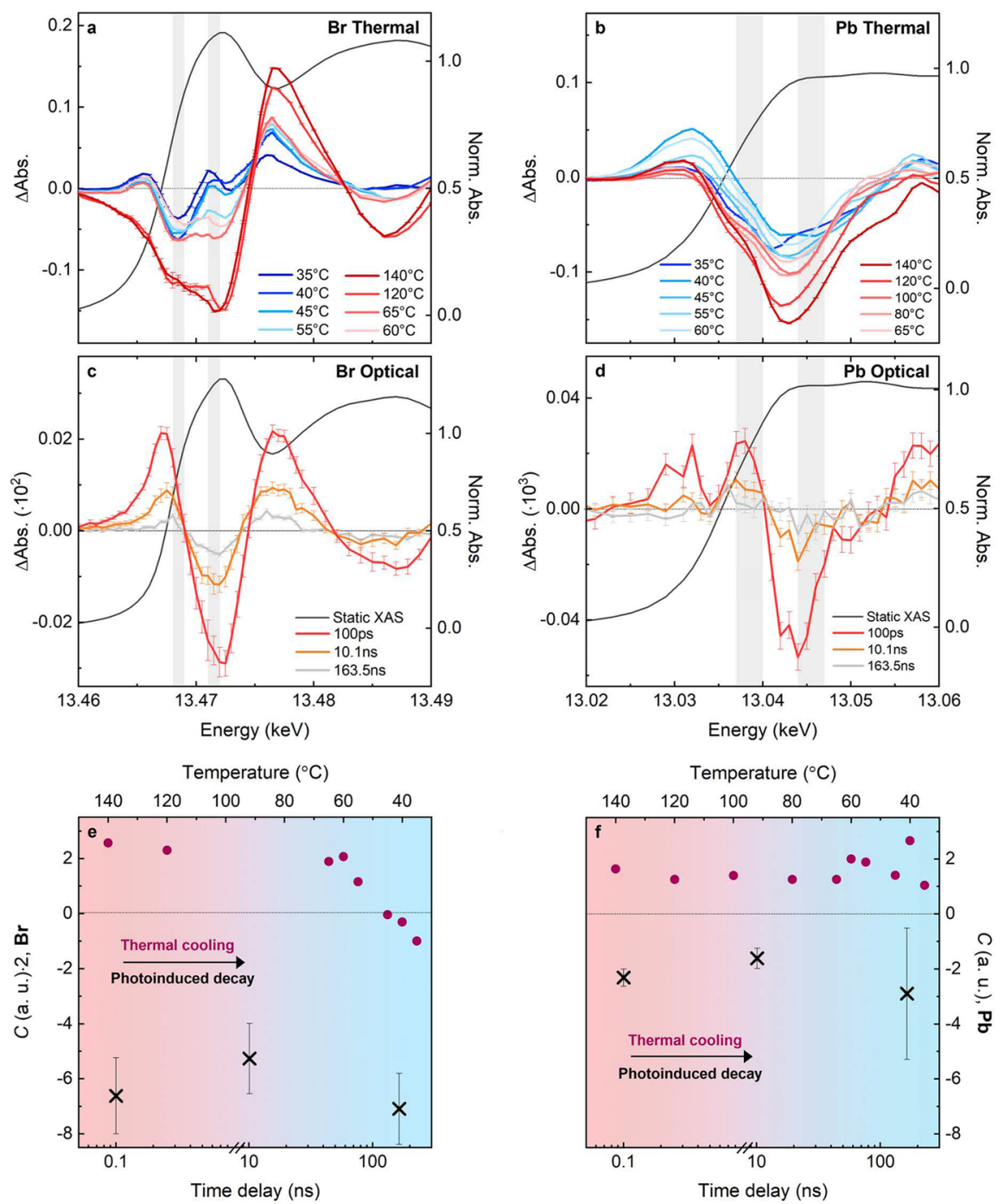

Figure 3. Comparison between photoinduced and thermally activated XAS transition in $\mathrm{CsPbBr}_{3}$ at the $\mathrm{Br} \mathrm{K}$-edge and $\mathrm{Pb} \mathrm{L}_{3}$-edge. (a) Br K-edge steady-state (dark gray) and temperature-dependent XAS differences from 35 to $140{ }^{\circ} \mathrm{C}$ (respectively from blue to red) and (b) $\mathrm{Pb} \mathrm{L}_{3}$-edge steadystate (dark gray) and temperature-dependent XAS differences from 35 to $140{ }^{\circ} \mathrm{C}$ (respectively from blue to red). In both panels, the XAS differences were computed by subtracting the $25^{\circ} \mathrm{C}$ spectrum from the temperature-dependent XAS spectra. Before performing the differences, all steady-state temperature-dependent spectra were baseline corrected and scaled by their underlying areas (consistently with the data treatment of time-resolved spectra). A three-point adjacent averaging of the spectra was performed to better track the evolution of the spectral shape as a function of the temperature. (c) Br K-edge steady-state (dark gray) and pump-probe spectra at $100 \mathrm{ps}$ (red), $10.1 \mathrm{~ns}$ (yellow), and $163.5 \mathrm{~ns}\left(\right.$ gray) and (d) $\mathrm{Pb} \mathrm{L}_{3}$-edge steady-state (dark gray) and pump-probe spectra at $100 \mathrm{ps}$ (red), $10.1 \mathrm{~ns}$ (yellow), and $163.5 \mathrm{~ns}$ (gray). The steady-state spectra in panels (a), (c) and (b), (d) show the same spectral shapes, accounting for their different energy resolution. (e) Br K-edge comparison parameter $C$ as a function of the temperature (purple dots, top axis) and pump-probe time delay (black crosses, bottom axis), defined as the ratio of the averaged XAS difference in the energy interval $13.471-13.472 \mathrm{keV}$ and $13.468-13.469 \mathrm{keV}$, corresponding to the shaded areas in panels (a) and (c). (f) $\mathrm{Pb} \mathrm{L}_{3}$-edge comparison parameter $C$ as a function of the temperature (purple dots, top axis) and pump-probe time delay (black crosses, bottom axis), defined as the ratio of the averaged XAS difference in the energy interval 13.044-13.047 keV and 13.037-13.040 keV, corresponding to the shaded areas in panels (b) and (d). $\mathrm{Br} C$ values for both thermal and optical data sets were multiplied by a factor of 2 in order to enable a straightforward comparison between $\mathrm{Br}$ and $\mathrm{Pb}$ results in panels (e) and (f).

generally point to photoinduced structural changes (further details in the SI). We will address these later, using $a b$ initio calculations and demonstrating their connection with photo-

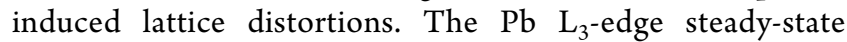
spectrum exhibits featureless edge and XANES regions. The transients are characterized by two positive features in the preedge region (13.031 and $13.038 \mathrm{keV})$, a negative peak at the edge position $(13.043 \mathrm{keV})$, and a positive peak in the postedge region $(13.060 \mathrm{keV})$. The reduction of $\mathrm{Pb}$ centers upon photoexcitation of the electrons in the $\mathrm{CB}$, which is mainly composed by $\mathrm{Pb}$ p orbitals, is not compatible with the transient line shape, as discussed by Santomauro et al. ${ }^{36}$ The appearance of pre-edge features in the transient traces can only be explained by the opening of new channels from the $2 p$ core orbitals. Indeed, due to hybridization, depleting the VB affects not only the $\mathrm{Br}$ centers but also the $\mathrm{Pb}$ ones, according to the computed p-DOS (see SI). Core-to-valence transitions can occur into the $\mathrm{Pb} 6$ s orbitals, which have a non-negligible contribution toward the top of the VB. Above the edge, the transient features are due to photoinduced structural changes. Because the $\mathrm{Pb}$ atoms are affected by the structural distortion around the Br centers (see 
below), it is likely that the above-edge features of the $\mathrm{Pb} \mathrm{L}_{3}$ absorption transient in part reflect the latter.

The decay kinetics at both $\mathrm{Br}$ and $\mathrm{Pb}$ main edges are shown in Figure $2 \mathrm{c}$. The traces were normalized to their maximum value, allowing a straightforward comparison of the time-resolved signal of both centers. It is clear that both traces show the same temporal evolution within the noise level. The data were analyzed following a global fit procedure for both traces. The best fit results were obtained with a biexponential decay function and a flat offset, which persists up to $130 \mathrm{~ns}$, the time limit explored in our time traces (see the SI). The fit function was convoluted with a Gaussian profile $(\sigma=45 \mathrm{ps})$, representing the instrument response function of our measurements. The recorded time constants (pre-exponential factors) are $\tau_{1}=120$ $\pm 20 \mathrm{ps}(60 \%), \tau_{2}=900 \pm 300 \mathrm{ps}(21 \%)$, and an offset (infinite times of $19 \%$ amplitude). Specifically, the fast time component $\tau_{1}$ is compatible with Auger recombination, where an electron in the $\mathrm{CB}$ and a hole in the $\mathrm{VB}$ recombine, in a nonradiative process, transferring their energy to a third carrier. Supporting this interpretation, recent fluence-dependent $\mathrm{PL}$ and transient absorption studies on $\mathrm{Cs} \mathrm{PbBr}_{3} \mathrm{NCs}$ reported Auger recombination acting on this time scale. ${ }^{52,53} \tau_{2}$ is ascribed to the radiative recombination of the photoexcited charge carriers, i.e., holes from the VB and electrons from the $\mathrm{CB}$, in general agreement with PL studies. ${ }^{44,54}$

Thermal XAS. Given the ongoing debate about photoinduced electronic and thermal effects ${ }^{37}$ and considering that our pump pulse deposits an excess energy of the photocarriers of $\sim 1 \mathrm{eV}$, it is important to disentangle electronic from thermal effects in the photoinduced response presented here.

In our pump-probe experiment, the hot carriers generated by the pumping process dissipate their excess energy through carrier thermalization in the sub-100 fs regime $^{21}$ and, immediately after, by charge carrier cooling on sub-picosecond time scales. ${ }^{55}$ These events determine impulsive heating of the crystalline lattice. If the energy deposited in the system is sufficiently high, this process might translate into impulsively activated orthorhombic-tetragonal-cubic phase transitions. ${ }^{13}$ At later time scales, the hot lattice relaxes through heat transfer to the solvent and/or the ligands. In ligand-stabilized colloidal NCs in solution this process should be completed in sub-nanosecond time scales, ${ }^{56}$ due to the efficient vibrational coupling between the NC, the ligands, and the solvent molecules. Our TR-XAS experiment looks at the system relaxation in time scales from 80 ps onward after photoexcitation, i.e., when the thermal equilibration of the lattice with the surrounding bath has already initiated. At these time scales, the photoinduced relaxation of the system and its purely thermal and temperature-dependent responses can be directly compared. This assumption can be harnessed to verify whether the optically induced relaxation coincides with thermodynamic lattice cooling.

Figure 3a,b show the thermal difference spectra at the $\mathrm{Br} \mathrm{K}$ and the $\mathrm{Pb} \mathrm{L}_{3}$-edges (full thermal spectra are available in the $\mathrm{SI}$ ). These are obtained by subtracting the $25^{\circ} \mathrm{C}$ spectrum from the $T$-dependent XANES spectra. Figures $3 \mathrm{c}, \mathrm{d}$ show the unscaled pump-probe difference spectra at 100 ps (red), $10.1 \mathrm{~ns}$ (yellow), and $163.5 \mathrm{~ns}$ (gray) for each absorption edge. The thermal difference spectra at the $\mathrm{Br}$ K-edge (Figure 3a) display an intensity change through the thermal gradient, with an overall area decrease in the energy range $13.466-13.478 \mathrm{keV}$ on lowering the temperature from $140{ }^{\circ} \mathrm{C}$ to $25^{\circ} \mathrm{C}$. Particularly, the features at 13.468 and $13.472 \mathrm{keV}$ have two different temperature dependences, the former becoming dominant for temperatures below $65{ }^{\circ} \mathrm{C}$. Main differences between photoinduced and thermal data sets can be found in the $\mathrm{Br} \mathrm{K}$ pre-edge region. Specifically, the negative feature at $13.468 \mathrm{keV}$ found in the thermal data set is absent in the pump-probe spectra, which instead are characterized by a positive peak centered around $13.4675 \mathrm{keV}$. In the case of $\mathrm{Pb}$, thermal difference spectra in Figure $3 \mathrm{~b}$ display a broad negative feature covering the 13.035$13.053 \mathrm{keV}$ spectral range, whose intensity uniformly decreases on lowering the temperature, and a single pre-edge feature centered at $13.032 \mathrm{keV}$, with intensity increasing upon temperature decrease. In contrast and as already discussed, the pump-probe $\mathrm{Pb}$ spectra show two pre-edge peaks, respectively at 13.031 and $13.038 \mathrm{keV}$, whose intensity decreases as a function of time delay.

To quantify overall spectral changes as a function of either temperature or optical excitation, at both edges, we introduce a comparison parameter $C$ defined as

$$
C=\sum_{E_{i}} I\left(E_{i}\right) / \sum_{E_{j}} I\left(E_{j}\right)
$$

with $I\left(E_{i}\right)$ and $I\left(E_{j}\right)$ being the spectral intensities at the energy points $E_{i}$ and $E_{j}$ in the main-edge and pre-edge regions, respectively. In Figure $3 \mathrm{a}-\mathrm{d}$ (gray areas), $E_{i}(\mathrm{Br})=13.471-$ $13.472 \mathrm{keV} ; E_{j}(\mathrm{Br})=13.468-13.469 \mathrm{keV} ; E_{i}(\mathrm{~Pb})=13.044-$ $13.047 \mathrm{keV}$; and $E_{j}(\mathrm{~Pb})=13.037-13.040 \mathrm{keV}$. The indicated intervals comprise equally spaced energy points. Figure $3 e, f$ show the parameter $\mathrm{C}$ for $\mathrm{Br}$ and $\mathrm{Pb}$, respectively: it expresses the relative intensity ratio between two spectral regions of the same data set, and it describes the entity of XAS spectral shape changes through a temperature gradient (purple dots) or upon photoexcitation (black crosses).

Within the error bars, $C$ values are constant in the pumpprobe case for either $\mathrm{Br}$ or $\mathrm{Pb}$, in agreement with the spectral evolution of the TR-XAS signal. Indeed, we observe a synchronous systems's response throughout the TR-XAS spectrum in its decay to the ground state. We remark that negative values of $C$ are due to the presence of the pre-edge and main-edge features that have opposite signs, respectively, at the energies 13.4675 and $13.472 \mathrm{keV}$ for the $\mathrm{Br} \mathrm{K}$-edge and at 13.038 and $13.044 \mathrm{keV}$ for the $\mathrm{Pb} \mathrm{L}_{3}$-edge. Starting from $140^{\circ} \mathrm{C}$, the $C$ parameter for the $\mathrm{Br}$ thermal differences has stable positive values for temperatures down to $60{ }^{\circ} \mathrm{C}$ and undergoes a progressive change from positive to negative values on lowering the temperature in the interval $60-35{ }^{\circ} \mathrm{C}$, due to line shape modifications in the edge region. Instead, $\mathrm{Pb}$ thermal differences show positive $C$ values at all temperatures, which originate from the negative sign of pre-edge and main-edge features. Since both features decrease in amplitude upon temperature decrease, $\mathrm{Pb} C$ values remain essentially unchanged with the thermal gradient.

On the basis of the radically different behavior of the $C$ parameter for the optical and thermal data sets, we can safely conclude that the photoresponses at the $\mathrm{Pb}$ and $\mathrm{Br}$ edges reported here are not affected by thermal effects, which likely occur on shorter time scales than our temporal window, as discussed in the following. Thus, we rule out the hypothesis that the photoexcited state corresponds to thermally driven lattice changes. $^{37}$

Theoretical Simulations: Photoinduced Polaronic Distortion. The XAS spectrum reflects the probability of an electronic excitation from a core orbital to the unoccupied states of the system that lie at higher energies than the Fermi level. 

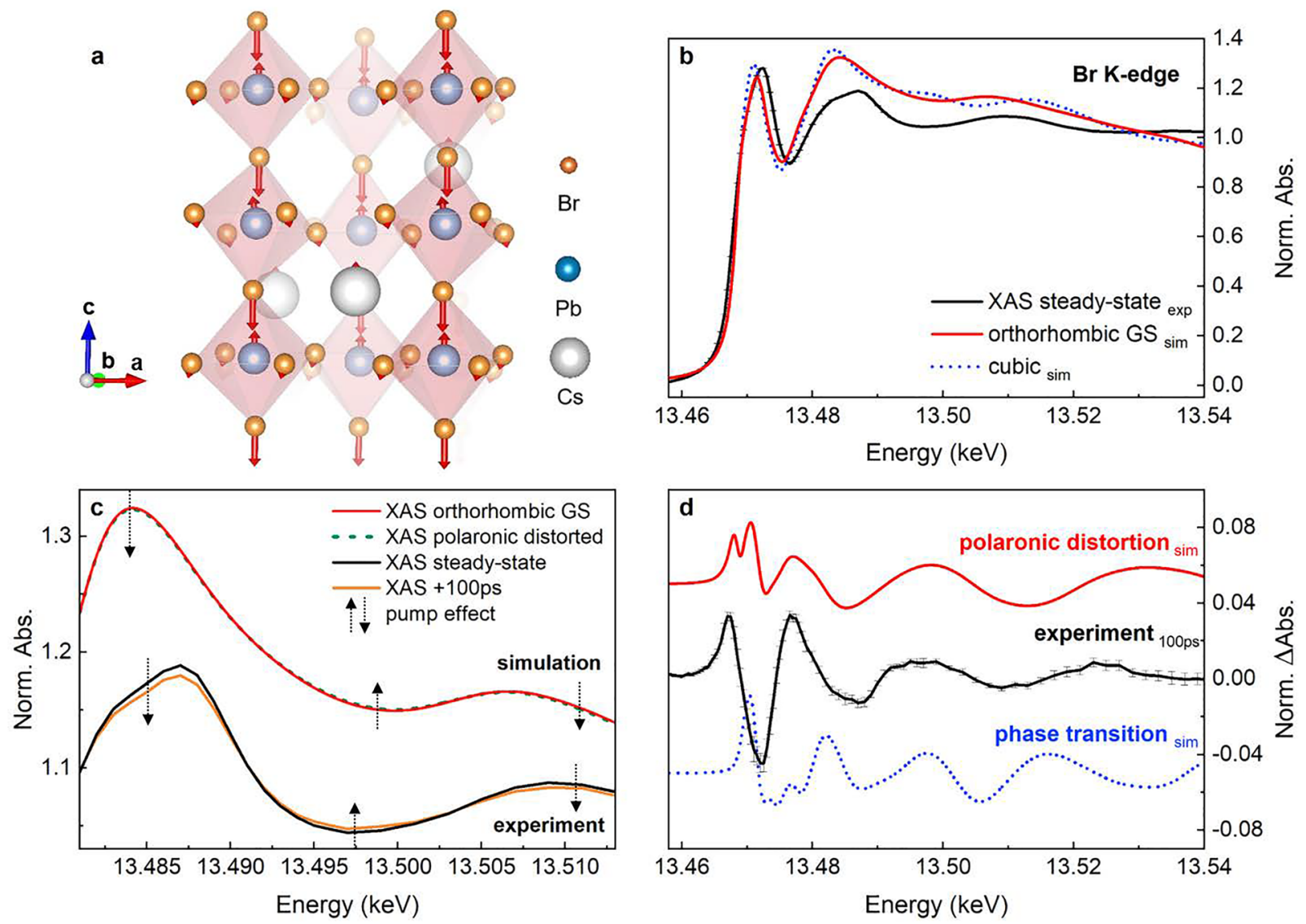

Figure 4. Theoretical ab initio calculations of $\mathrm{Br}$ K-edge XAS spectra for the ground and structurally distorted states in $\mathrm{CsPbBr}_{3}$. (a) $\mathrm{Schematics}_{\mathrm{s}}$ of the atomic displacements related with the $18 \mathrm{meV}$ LO phonon mode. (b) Steady-state experiment (black, normalized for the last energy point), groundstate orthorhombic simulation (red solid line, scaling factor $\times 2550$ ), and cubic simulation (blue dashed line, scaling factor $\times 2550)$. $(\mathrm{c})$ Ground-state orthorhombic simulation (red, scaling factor $\times 2550$ ), orthorhombic distorted along the $18 \mathrm{meV}$ LO phonon mode (green dashed line, scaling factor $\times 2550$ ), and unpumped (black, normalized for the last energy point) and pumped (yellow, 100 ps time delay, normalized for the last energy point) experimental spectra. (d) Experimental transient at $100 \mathrm{ps}$ (black) and simulated pump-probe obtained as distorted orthorhombic minus pristine orthorhombic spectra (polaronic distortion, red) and cubic minus orthorhombic spectra (phase transition, blue dashed line). All spectra were scaled by the absolute area underlying the curves and the simulated pump-probe additionally multiplied by a factor 70 to enable the comparison with the experiment.

Hence, the first-principles description of XANES spectra requires the computation of highly localized initial orbitals and of the unoccupied conduction states, the latter in the presence of a screened core-hole, since it reflects the possible final states with a missing core electron.

In condensed matter systems, this level of accuracy is retrieved relying on band structure calculations, where the effect of the screened core-hole is explicitly accounted for as, for example, in supercell simulations. ${ }^{57,58}$ Band structure calculations also allow access to the phonon spectrum of the system, the atomic displacements occurring upon phonon mode activation, and the electron-phonon coupling between the charge carriers and the lattice degrees of freedom. To date XAS spectra of several solidstate systems have been computed using $a b$ initio methods, which rely on real-space atomic clusters, especially for the simulation of excited-state spectra. ${ }^{32,59-61}$ In these cases, the interest was focused on determining the local structural distortions in the photoexcited system, as in the case of charge carriers trapping, rather than understanding the origin of its structural response in the presence of charge carriers, which requires electron-phonon coupling calculations.

First-principles computations of pump-probe spectra are generally performed either using a-posteriori strategies, i.e., gradually modifying the local structure of a small atomic cluster until the best agreement between the simulation and the experiment is achieved, ${ }^{32,59,61}$ or selecting a-priori specific subsets of configurations, where stricter constraints are imposed on a physical basis. ${ }^{60}$ Here, to our knowledge for the first time in TR-XAS, we adopt an approach based on $a b$ initio calculations performed under periodic boundary conditions with an a-priori selection of the structurally distorted states. Consistent with the phase diagram of $\mathrm{CsPbBr}_{3}$ perovskite, ${ }^{13,16}$ we computed the ground-state considering the atomic positions of the Pnma orthorhombic cell, as derived from room-temperature XRD. ${ }^{16}$ The lattice perturbation caused by the optical pump was then simulated following two possible scenarios.

First we consider the scenario, ruled out experimentally, in which a thermally induced phase transition to an ordered $P m \overline{3} m$ cubic state might occur. In fact, we estimated an upper limit of $\Delta T \approx 120^{\circ} \mathrm{C}$ to the impulsive heating generated by our pump pulse (see the SI), which, in the absence of lattice cooling and consistent with the $\mathrm{Cs} \mathrm{PbBr}_{3}$ phase diagram, would lead the system from the orthorhombic to the cubic phase. For our simulation we use the atomic coordinates available in the literature. ${ }^{16}$ Second, we consider a polaronic distortion induced by electron-hole pair excitation, introducing a structural 
modification along the $18 \mathrm{meV}$ phonon mode, which is the most strongly coupled to the charge carriers via electron-phonon coupling. ${ }^{38}$ The structural modification caused by the aforementioned process is schematized in Figure 4a. Key to this method is the adoption of a band structure calculation, which allows (i) identifying the phonon mode with the strongest electron-phonon coupling and (ii) introducing the phonon distortion in the periodic lattice, thereby approximating the large polaron spatial extension over multiple unit cells, in agreement with the literature. ${ }^{14,38}$ Electronic nonequilibrium effects following the optical excitation were not included in the calculations, being too computationally intensive in the presence of an explicit core-hole description.

Figure $4 \mathrm{~b}$ compares the simulated $\mathrm{Br}$ K-edge absorption spectra for the orthorhombic ground state (red solid) and the cubic state (blue dots) with the experimental steady-state spectrum (black solid). The three X-ray absorption spectra are characterized by a first peak arising from the $\mathrm{Br} 1 \mathrm{~s}-4 \mathrm{p}$ electronic transition around $13.472 \mathrm{keV}$. The above-edge spectral modulations for the calculated orthorhombic ground state best reproduce the experiment, as expected from the $\mathrm{CsPbBr}$ perovskite phase diagram, which is characterized by a Pnma orthorhombic symmetry at room temperature. The simulated spectrum for the cubic phase shows a modulation mismatch with respect to the experiment, which is prominent in the 13.492$13.505 \mathrm{keV}$ energy range.

Figure $4 \mathrm{c}$ zooms into the above-edge region beyond 13.480 $\mathrm{keV}$ of the experimental pumped and unpumped spectra (respectively yellow and black traces) and for the simulated orthorhombic ground state and the polaronic distorted state (respectively red and dashed-green traces). Even though the photoinduced changes are more pronounced in the experiment, the simulation faithfully follows the photoinduced spectral modification, with intensity depletions of the 13.485 and 13.508 $\mathrm{keV}$ maxima and an intensity increase of the $13.497 \mathrm{keV}$ minimum.

Figure $4 \mathrm{~d}$ shows the simulated pump-probe signals obtained subtracting the XAS spectrum of the orthorhombic ground state from the XAS spectrum due to the polaronic distortion (red solid) as well as from the cubic phase XAS spectrum (blue dots). The comparison of the difference curves allows removing possible systematic errors of our $a b$ initio calculation for both ground and excited states. Above the edge, the experimental pump-probe spectrum at $100 \mathrm{ps}$ shows very good agreement in both position and relative amplitude assuming a polaronic lattice distortion generated by the optical activation of the $18 \mathrm{meV} \mathrm{LO}$ phonon within the polar inorganic lattice. On the other hand, there is a clear disagreement with the simulation that assumes the phase transition to the $P m \overline{3} m$ cubic structure. We highlight that the XAS simulation for the orthorhombic-cubic phase transition does not reproduce the spectral line shape of the 120 ${ }^{\circ} \mathrm{C}$ minus $25^{\circ} \mathrm{C}$ thermal difference reported in Figure 3a either. This result is analyzed in a separate work.

We remark that the residual deviations between the simulated polaronic distortion and the experimental pump-probe spectra (Figure 4d) can be rationalized considering that electronic effects caused by the optical pump are absent in the calculation. Indeed, the edge region is particularly sensitive to photoinduced changes of the unoccupied DOS of the system. Relying on the one-electron approximation, in $\mathrm{Br}$ K-edge transitions the initial and final states differ by the presence of a core-hole in a $1 \mathrm{~s} \mathrm{Br}$ orbital and a photoelectron above the Fermi level. Due to the localization of the $1 \mathrm{~s}$ orbitals on $\mathrm{Br}$ atoms, the $\mathrm{Br}$ K-edge transition probability is non-negligible only for final states where the photoelectron has a significant character of the $\mathrm{Br}$ absorbing atom, which are present in both the VB and CB (see the SI). When the band occupancy is perturbed by the optical pump, the $\mathrm{Br}$ XANES in the edge region is also modified.

\section{DISCUSSION}

The comparison of optical XAS study with temperaturedependent XAS measurements rules out a dominant photoinduced thermal effect in the TR-XAS response. Indeed, if the evolution of the pump-probe spectral line shape reflected the lattice cooling following impulsive heating, a change in the TRXAS signal intensity and line shape similar to Figure $3 a, b$ should be expected. However, this is not observed in the transients reported in Figure 3c,d. Furthermore, this discrepancy is confirmed by the differences in the $C$ parameters of Figure $3 e, f$ between the thermal and optical data sets. Thus, even though a significant heat deposition could occur under our experimental conditions (see the SI for calculations), a thermal origin of the transient signal can hardly justify the strong difference between the time evolution of the pump-probe signal and the changes expected for thermal cooling.

In ligand-stabilized colloidal NCs, heat transport is known to be determined by the organic/inorganic interface rather than the thermal conductivity of the inorganic core of the NC. ${ }^{62}$ In CdSe $\mathrm{NC}$ systems, the heat loss from the ligand-NC complex to the bath was observed in 150-320 ps, depending on the solvent. ${ }^{56}$ Considering the similarity of the ligand-capped CdSe system and our NCs in size, ligand composition, low-energy NC phonon spectrum, fast thermalization dynamics, ${ }^{56,63}$ and the main role of the ligand-solvent coupling to the cooling process, analogous time scales are expected for our $\mathrm{CsPbBr}_{3} \mathrm{NCs}$ in solution. Relying on Newton's law to describe the NC lattice cooling and assuming a relatively slow $\tau_{\text {cooling }}$ of $300 \mathrm{ps,} \mathrm{an} \mathrm{initial}$ $\Delta T \approx 120^{\circ} \mathrm{C}$ temperature would quickly drop to smaller values, e.g., $\mathrm{T}_{\mathrm{NC}}(t=600 \mathrm{ps}) \approx 40^{\circ} \mathrm{C}$.

This prediction is in stark contrast with the persistency of the TR-XAS signal over time, which preserves the same line shape at the $\mathrm{Br}$ and $\mathrm{Pb}$ edges up to the longest time delay measured in our pump-probe experiment, namely, $163.5 \mathrm{~ns}$. We conclude that heat dissipation in zwitterion-capped $\mathrm{Cs}_{\mathrm{PbBr}}$ perovskite NCs should be complete in shorter time scales than our TR-XAS time resolution, not affecting the pump-probe measurements. Notably, similar results were reported in a $\mathrm{Pb} \mathrm{L}_{3}$-edge TRXAS investigation on $\mathrm{MAPbBr}_{3}$ ligand-capped NCs in solution, ${ }^{32}$ where the significant heat load caused by the pump energy deposition into the NC lattice was argued to be dissipated in time scales shorter than 100 ps.

The light-activated structural modification is not compatible with a cubic crystalline structure, nor is it due to disorder, amorphization, or melting caused by thermal effects. Our theoretical analysis clarifies key aspects of the photoinduced response of $\mathrm{CsPbBr}_{3}$ perovskite NCs, ascribing the excited-state structural changes to the presence of distinct polaronic distortions that the XAS simulation specifically identifies. Indeed, the atomic displacements of the $\mathrm{Pb}-\mathrm{Br}$ framework are traced back to the distortion of the $18 \mathrm{meV}$ LO phonon mode, which is the most strongly responsive to the charge carriers via electron-phonon coupling. Strong electron-phonon coupling in lead halide perovskites was demonstrated in the electronic structure of $\mathrm{Cs} \mathrm{PbBr}_{3}$ single crystals, where signatures of large hole polarons were identified by ARPES and attributed to the activation of the same LO phonon mode. ${ }^{38}$ Moreover, previous 
time-domain results based on the optical Kerr effect, ${ }^{14}$ electronic resonant and nonresonant impulsive vibrational spectroscopy, ${ }^{25}$ ultrafast $\mathrm{THz}$ studies, ${ }^{27}$ and $2 \mathrm{D}$ electronic spectroscopy ${ }^{24}$ were rationalized in terms of polaron formation in organic and inorganic perovskites. Polaronic strain was also invoked as the primary driving force of light-induced phase separation in multihalide perovskites, explaining the reversibility of the process, its dependence on the number of photocarriers, and the selflimiting size of the domains. ${ }^{64}$ The importance of electronphonon coupling on the $\mathrm{Cs} \mathrm{PbBr}_{3}$ electronic response was also confirmed by PL investigations ${ }^{65}$ and time-resolved $2 \mathrm{D}$ electronic spectroscopy, ${ }^{26}$ pointing to a relevant influence of LO phonon modes with energies between 16 and $19 \mathrm{meV}$, and attributed to the lead halide inorganic framework, consistently with our findings.

Thanks to the agreement with the TR-XAS experimental results, our simulations provide a compelling atomic-level description of the polaronic distortion. As depicted in Figure $4 \mathrm{a}$, the distortion along the $18 \mathrm{meV}$ phonon mode implies that the $\mathrm{Pb}-\mathrm{Br}$ bonds are asymmetrically shortened along the orthorhombic $c$-axis, moving $\mathrm{Pb}$ cations out of the octahedral inversion center and substantially displacing the axial $\mathrm{Br}$ nuclei from their equilibrium position, whereas the equatorial $\mathrm{Br}$ centers and the Cs ions are marginally affected. Specifically, the photoinduced displacement of the axial $\mathrm{Br}$ atoms along the $c$-axis $\left(\mathrm{Pb}-\mathrm{Br}_{\text {axial }}\right.$ equilibrium bond distance $\left.=2.958 \AA\right)$ is 6 times larger than the equatorial $\mathrm{Br}$ atoms $\left(\mathrm{Pb}-\mathrm{Br}_{\text {equatorial }}\right.$ equilibrium bond distance $=2.964 \AA$ ) and 2.5 times more pronounced than the $\mathrm{Pb}$ off-center displacement. The postedge modulations observed in the $\mathrm{Pb} \mathrm{L}_{3}$-edge transients can thus be explained by the displacements of the $\mathrm{Pb}$ and $\mathrm{Br}$ centers caused by the photogenerated polaronic distortion. Notably, the previously reported absence of a photoinduced structural response from the Cs centers ${ }^{36}$ also agrees with this finding. Indeed, in allinorganic perovskites the $\mathrm{A}^{+}$cation allocated in the lattice cuboctahedral voids is largely mobile and its dynamics is essentially decoupled from the inorganic $\mathrm{Pb}-\mathrm{X}$ framework. ${ }^{10}$ Further supporting the above description is that the kinetic traces and transient XAS energy profiles point to a concerted behavior of $\mathrm{Br}$ and $\mathrm{Pb}$ in response to the optical excitation. The time scales of the intensity decays are fully in line with the Auger $^{52,53}$ and PL recombination lifetimes ${ }^{44,54}$ in $\mathrm{CsPbBr}_{3}$ perovskites. The relaxation occurs with a direct recovery of the perovskite's ground state, as confirmed by the retention of the TR-XAS line shapes in the decay process and by the time evolution of the $C$ parameter.

The high PL quantum yields reported for $\mathrm{CsPbBr}_{3} \mathrm{NCs}^{44}$ and the fact that the transients do not change line shape profile, i.e., there is no evidence for intermediate states, point to a recovery of the system largely dominated by charge carrier recombination. The presence of polaronic distortions is consistent with this scenario: after the photocarriers have induced the lattice displacements dictated by the strong electron-phonon coupling of the system, the subsequent electron-hole recombination causes the reversible unlocking of the structural distortions of the $\mathrm{Pb}-\mathrm{Br}$ framework, back to the ground-state configuration.

Notably, spectral line changes as a function of time delay were detected in $\mathrm{Cs}_{3} \mathrm{Bi}_{2} \mathrm{Br}_{9}$ perovskites with $\mathrm{Br} \mathrm{K}$-edge TR-XAS as a consequence of their asynchronous electronic and structural relaxation upon optical excitation, ${ }^{59}$ with long-persisting lattice disorder after charge carrier recombination. The observation of short-lived valence holes in $\mathrm{Cs}_{3} \mathrm{Bi}_{2} \mathrm{Br}_{9}$, compared to the postedge signatures of lattice distortion, indicates that composition and structure of the inorganic sublattice in halide perovskites, either $\mathrm{Bi}_{2} \mathrm{Br}_{9}$ or $\mathrm{PbBr}_{3}$, can strongly influence the photodynamics of the system and thus its optoelectronic performances.

This work also highlights the importance of local structural techniques in unraveling the nature of electronic and structural changes in perovskites, triggered by different external perturbations. In diffraction, structural modifications are obtained using approaches that go beyond standard Rietveld refinement methods. One of these methods relies, for example, on the computation of the Fourier transform of the total scattering structure factor to retrieve the PDF, which expresses a probability of finding pairs of atoms separated by a distance $r .{ }^{66}$ $\mathrm{PDF}$ analysis from X-ray powder diffraction in a host of organic perovskites showed significant internal local distortions of the lead halide octahedra at room temperature. ${ }^{19}$ Later, total scattering structural characterization, relying on a joint Debye scattering equation/atomic PDF approach, clarified that in $\mathrm{CsPbBr}_{3} \mathrm{NCs}$ the structural defectiveness is due to twin boundaries, whose density increase with temperature leads to an apparent higher-symmetry structure that does, however, not correspond to the $P m \overline{3} m$ cubic phase. ${ }^{12}$ A recent high energy resolution inelastic X-ray scattering and $\mathrm{PDF}$ study on $\mathrm{MAPbI}_{3}$ pointed to the presence of thermally active anharmonic soft modes at $350 \mathrm{~K}$, corresponding to in-phase and out-of-phase rotations of the $\mathrm{PbI}_{6}$ octahedra. ${ }^{11}$ Shortly after, local polar fluctuations were also confirmed in $\mathrm{MAPbBr}_{3}$ and $\mathrm{CsPbBr}$ perovskites in a temperature-dependent Raman study, where the presence of a zero-frequency Raman peak was assigned to anharmonic thermal fluctuations among different noncubic structures. $^{15}$

All these studies underline that correlating medium- to longrange structural methods with local probes helps distinguish subtle changes in the perovskite lattice. In this respect, XAS represents a correlative short-range structural tool to probe disordered or dynamically changing systems such as lead halide perovskite NCs. In its time-resolved implementation, TR-XAS offers the advantage of combining electronic and local structural sensitivity, making it an ideal technique to probe lattice modifications induced by the presence of photocarriers, as in the case of polaron formation ${ }^{32,33,36,59}$ or charge trapping, ${ }^{60,67}$ and to discern them from thermally induced changes.

\section{CONCLUSIONS}

We presented results of light- and temperature-induced changes at the $\mathrm{Br}$ K-edge and $\mathrm{Pb} \mathrm{L}_{3}$-edge of $\mathrm{CsbBr}_{3} \mathrm{NCs}$ dispersed in toluene solution or as dry powders. Our findings show strong differences between the thermal and optical response of the system, excluding dominant photothermal effects in the observed pump-probe dynamics. The photoinduced spectral changes at the $\mathrm{Br}$ K-edge, stemming from a polaron distortion, are here quantified for the first time using advanced band structure calculation, including an a-priori selection of the excited state and fully accounting for core-hole effects on the TR-XAS spectra. The comparison between our experiment and theory identifies the lattice changes at the origin of the transient $\mathrm{Br}$ postedge modulations with a distortion along an $\mathrm{LO}$ phonon mode at $18 \mathrm{meV}$. These simulations provide an atomic-level description of the light-induced nuclear displacement, dominated by an asymmetric $\mathrm{Pb}-\mathrm{Br}$ bond shortening along the orthorhombic $c$-axis. This is supported by the identical kinetic evolution of the transient $\mathrm{Br} \mathrm{K}$-edge and $\mathrm{Pb} \mathrm{L}_{3}$-edge transients, which show that the latter is a direct consequence of the polaronic distortion around $\mathrm{Br}$ centers. This is also consistent 
with the high $\mathrm{PL}$ quantum yields reported for $\mathrm{CsPbBr}_{3} \mathrm{NCs}^{44}$ and unravels new microscopic insights in the $\mathrm{Pb}-\mathrm{Br}$ sublattice dynamics, clarifying the perovskite response under light-induced out-of-equilibrium conditions.

\section{ASSOCIATED CONTENT}

\section{SI Supporting Information}

The Supporting Information is available free of charge at https://pubs.acs.org/doi/10.1021/jacs.1c02403.

Samples and characterization, experimental methods, fluence scans data analysis, energy scans data analysis, time scans data analysis, estimation of the number of photocarriers, photoinduced blue shift of the Br K-edge, computational methods and DOS calculation, T-dependent XRD and XAS (PDF)

\section{AUTHOR INFORMATION}

\section{Corresponding Authors}

Majed Chergui - Laboratory of Ultrafast Spectroscopy (LSU) and Lausanne Centre for Ultrafast Science (LACUS), Ecole Polytechnique Fédérale de Lausanne, CH-1015 Lausanne, Switzerland; 이이이.org/0000-0002-4856-226X; Email: majed.chergui@epfl.ch

Giulia F. Mancini - Laboratory of Ultrafast Spectroscopy (LSU) and Lausanne Centre for Ultrafast Science (LACUS), Ecole Polytechnique Fédérale de Lausanne, CH-1015 Lausanne, Switzerland; Present Address: Department of Physics, University of Pavia, I-27100 Pavia, Italy.; () orcid.org/0000-0002-7752-2822;

Email: giuliafulvia.mancini@unipv.it

\section{Authors}

Oliviero Cannelli - Laboratory of Ultrafast Spectroscopy (LSU) and Lausanne Centre for Ultrafast Science (LACUS), Ecole Polytechnique Fédérale de Lausanne, CH-1015 Lausanne, Switzerland; 이이.org/0000-0002-1844-4799

Nicola Colonna - Laboratory for Neutron Scattering and Imaging, Paul Scherrer Institute, CH-5232 Villigen-PSI, Switzerland; National Centre for Computational Design and Discovery of Novel Materials (MARVEL), École Polytechnique Fédérale de Lausanne, CH-1015 Lausanne, Switzerland; (1) orcid.org/0000-0002-6106-6316

Michele Puppin - Laboratory of Ultrafast Spectroscopy (LSU) and Lausanne Centre for Ultrafast Science (LACUS), Ecole Polytechnique Fédérale de Lausanne, CH-1015 Lausanne, Switzerland; (1) orcid.org/0000-0002-1328-7165

Thomas C. Rossi - Laboratory of Ultrafast Spectroscopy (LSU) and Lausanne Centre for Ultrafast Science (LACUS), Ecole Polytechnique Fédérale de Lausanne, CH-1015 Lausanne, Switzerland; Present Address: Department of Chemistry and Materials Research Laboratory, University of Illinois at Urbana-Champaign, Champaign, Illinois 61820, United States.; (1) orcid.org/0000-0002-7448-8948

Dominik Kinschel - Laboratory of Ultrafast Spectroscopy (LSU) and Lausanne Centre for Ultrafast Science (LACUS), Ecole Polytechnique Fédérale de Lausanne, CH-1015 Lausanne, Switzerland; 이이.org/0000-0002-0269-8567

Ludmila M. D. Leroy - Laboratory of Ultrafast Spectroscopy (LSU) and Lausanne Centre for Ultrafast Science (LACUS), Ecole Polytechnique Fédérale de Lausanne, CH-1015 Lausanne, Switzerland; LabCri, Universidade Federal de
Minas Gerais, 31270-901 Belo Horizonte, Brazil; (1) orcid.org/0000-0002-4272-0298

Janina Löffler - Laboratory of Ultrafast Spectroscopy (LSU) and Lausanne Centre for Ultrafast Science (LACUS), Ecole Polytechnique Fédérale de Lausanne, CH-1015 Lausanne, Switzerland; 이이.org/0000-0003-3147-8640

James M. Budarz - Laboratory of Ultrafast Spectroscopy (LSU) and Lausanne Centre for Ultrafast Science (LACUS), Ecole Polytechnique Fédérale de Lausanne, CH-1015 Lausanne, Switzerland; ○ orcid.org/0000-0002-7673-6626

Anne Marie March - Chemical Sciences and Engineering Division, Argonne National Laboratory, Lemont, Illinois 60439, United States; 이이.org/0000-0003-2961-1246

Gilles Doumy - Chemical Sciences and Engineering Division, Argonne National Laboratory, Lemont, Illinois 60439, United States; orcid.org/0000-0001-8672-4138

Andre Al Haddad - Chemical Sciences and Engineering Division, Argonne National Laboratory, Lemont, Illinois 60439, United States; Present Address: Paul Scherrer Institute (PSI), 5232 Villigen, Switzerland.

Ming-Feng Tu - Chemical Sciences and Engineering Division, Argonne National Laboratory, Lemont, Illinois 60439, United States; orcid.org/0000-0001-7834-0329

Yoshiaki Kumagai - Chemical Sciences and Engineering Division, Argonne National Laboratory, Lemont, Illinois 60439, United States; 이이이.org/0000-0002-2492-4676

Donald Walko - Advanced Photon Source, Argonne National Laboratory, Lemont, Illinois 60439, United States

Grigory Smolentsev - Paul Scherrer Institute (PSI), 5232 Villigen, Switzerland; ○ orcid.org/0000-0001-7348-7276

Franziska Krieg - Institute of Inorganic Chemistry, Department of Chemistry and Applied Biosciences, ETH Zürich, CH-8093 Zürich, Switzerland; Laboratory for Thin Films and Photovoltaics, Empa-Swiss Federal Laboratories for Materials Science and Technology, CH-8600 Dübendorf, Switzerland; ○ orcid.org/0000-0002-0370-1318

Simon C. Boehme - Institute of Inorganic Chemistry, Department of Chemistry and Applied Biosciences, ETH Zürich, CH-8093 Zürich, Switzerland; Laboratory for Thin Films and Photovoltaics, Empa-Swiss Federal Laboratories for Materials Science and Technology, CH-8600 Dübendorf, Switzerland; 이이.org/0000-0002-8399-5773

Maksym V. Kovalenko - Institute of Inorganic Chemistry, Department of Chemistry and Applied Biosciences, ETH Zürich, CH-8093 Zürich, Switzerland; Laboratory for Thin Films and Photovoltaics, Empa-Swiss Federal Laboratories for Materials Science and Technology, CH-8600 Dübendorf, Switzerland; 이이이.org/0000-0002-6396-8938

Complete contact information is available at:

https://pubs.acs.org/10.1021/jacs.1c02403

\section{Notes}

The authors declare no competing financial interest.

Processed data shown in this manuscript are available in the Supporting Information. Raw $\mathrm{Br}$ K-edge and $\mathrm{Pb} \mathrm{L}_{3}$-edge XAS data were generated at APS and SLS large-scale facilities and are available in the repository 10.5281 /zenodo.4564629.

\section{ACKNOWLEDGMENTS}

This work was supported by the European Research Council Advanced Grant H2020 ERCEA 695197 DYNAMOX and by the SwissNSF NCCR-MUST and NCCR-MARVEL. G.F.M. 
acknowledges the support of the European Union's Horizon 2020 research and innovation program, through the grant agreement no. 851154 (ULTRAIMAGE). The Argonne group (G.D., A.M.M., A.A., Y.K., M.-F.T.) was supported by the U.S. Department of Energy, Office of Science, Basic Energy Sciences, Chemical Sciences, Geosciences, and Biosciences Division, under contract DE-AC02-06CH11357. This research used resources of the Advanced Photon Source, a U.S. Department of Energy (DOE) Office of Science User Facility operated for the DOE Office of Science by Argonne National Laboratory under Contract No. DE-AC02-06CH11357. M.K. acknowledges funding by the European Union's Horizon 2020 program, through a FET Open research and innovation action under the grant agreement no. 899141 (PoLLoC). S.C.B. acknowledges The Netherlands Organization of Scientific Research (NWO) for financial support through the Innovational Research Incentives (Veni) Scheme (722.017.011).

\section{REFERENCES}

(1) Liu, X.-K.; Xu, W.; Bai, S.; Jin, Y.; Wang, J.; Friend, R. H.; Gao, F. Metal Halide Perovskites for Light-Emitting Diodes. Nat. Mater. 2021, $20,1-12$.

(2) Sutherland, B. R.; Sargent, E. H. Perovskite Photonic Sources. Nat. Photonics 2016, 10 (5), 295-302.

(3) Fang, Y.; Dong, Q.; Shao, Y.; Yuan, Y.; Huang, J. Highly Narrowband Perovskite Single-Crystal Photodetectors Enabled by Surface-Charge Recombination. Nat. Photonics 2015, 9 (10), 679-686.

(4) Su, R.; Ghosh, S.; Wang, J.; Liu, S.; Diederichs, C.; Liew, T. C. H.; Xiong, Q. Observation of Exciton Polariton Condensation in a Perovskite Lattice at Room Temperature. Nat. Phys. 2020, 16 (3), 301-306.

(5) Utzat, H.; Sun, W.; Kaplan, A. E. K.; Krieg, F.; Ginterseder, M.; Spokoyny, B.; Klein, N. D.; Shulenberger, K. E.; Perkinson, C. F.; Kovalenko, M. V.; Bawendi, M. G. Coherent Single-Photon Emission from Colloidal Lead Halide Perovskite Quantum Dots. Science 2019, 363 (6431), 1068-1072.

(6) Akkerman, Q. A.; Rainò, G.; Kovalenko, M. V.; Manna, L. Genesis, Challenges and Opportunities for Colloidal Lead Halide Perovskite Nanocrystals. Nat. Mater. 2018, 17 (5), 394-405.

(7) Xing, G.; Mathews, N.; Sun, S.; Lim, S. S.; Lam, Y. M.; Grätzel, M.; Mhaisalkar, S.; Sum, T. C. Long-Range Balanced Electron- and HoleTransport Lengths in Organic-Inorganic $\mathrm{CH}_{3} \mathrm{NH}_{3} \mathrm{PbI}_{3}$. Science 2013, 342 (6156), 344-347.

(8) Stranks, S. D.; Eperon, G. E.; Grancini, G.; Menelaou, C.; Alcocer, M. J. P.; Leijtens, T.; Herz, L. M.; Petrozza, A.; Snaith, H. J. ElectronHole Diffusion Lengths Exceeding 1 Micrometer in an Organometal Trihalide Perovskite Absorber. Science 2013, 342 (6156), 341-344.

(9) Herz, L. M. Charge-Carrier Dynamics in Organic-Inorganic Metal Halide Perovskites. Annu. Rev. Phys. Chem. 2016, 67 (1), 65-89.

(10) Miyata, K.; Atallah, T. L.; Zhu, X.-Y. Lead Halide Perovskites: Crystal-Liquid Duality, Phonon Glass Electron Crystals, and Large Polaron Formation. Sci. Adv. 2017, 3 (10), No. e1701469.

(11) Beecher, A. N.; Semonin, O. E.; Skelton, J. M.; Frost, J. M.; Terban, M. W.; Zhai, H.; Alatas, A.; Owen, J. S.; Walsh, A.; Billinge, S. J. L. Direct Observation of Dynamic Symmetry Breaking above Room Temperature in Methylammonium Lead Iodide Perovskite. ACS Energy Lett. 2016, 1 (4), 880-887.

(12) Bertolotti, F.; Protesescu, L.; Kovalenko, M. V.; Yakunin, S.; Cervellino, A.; Billinge, S. J. L.; Terban, M. W.; Pedersen, J. S.; Masciocchi, N.; Guagliardi, A. Coherent Nanotwins and Dynamic Disorder in Cesium Lead Halide Perovskite Nanocrystals. ACS Nano 2017, 11 (4), 3819-3831.

(13) Cottingham, P.; Brutchey, R. L. Depressed Phase Transitions and Thermally Persistent Local Distortions in $\mathrm{Cs} \mathrm{PbBr}_{3}$ Quantum Dots. Chem. Mater. 2018, 30 (19), 6711-6716.
(14) Miyata, K.; Meggiolaro, D.; Trinh, M. T.; Joshi, P. P.; Mosconi, E.; Jones, S. C.; Angelis, F. D.; Zhu, X.-Y. Large Polarons in Lead Halide Perovskites. Sci. Adv. 2017, 3 (8), No. e1701217.

(15) Yaffe, O.; Guo, Y.; Tan, L. Z.; Egger, D. A.; Hull, T.; Stoumpos, C. C.; Zheng, F.; Heinz, T. F.; Kronik, L.; Kanatzidis, M. G.; Owen, J. S.; Rappe, A. M.; Pimenta, M. A.; Brus, L. E. Local Polar Fluctuations in Lead Halide Perovskite Crystals. Phys. Rev. Lett. 2017, 118 (13), 136001.

(16) Stoumpos, C. C.; Malliakas, C. D.; Peters, J. A.; Liu, Z.; Sebastian, M.; Im, J.; Chasapis, T. C.; Wibowo, A. C.; Chung, D. Y.; Freeman, A. J.; Wessels, B. W.; Kanatzidis, M. G. Crystal Growth of the Perovskite Semiconductor $\mathrm{Cs}_{\mathrm{PbBr}_{3}}$ : A New Material for High-Energy Radiation Detection. Cryst. Growth Des. 2013, 13 (7), 2722-2727.

(17) Kovalenko, M. V.; Protesescu, L.; Bodnarchuk, M. I. Properties and Potential Optoelectronic Applications of Lead Halide Perovskite Nanocrystals. Science 2017, 358 (6364), 745-750.

(18) Fujii, Y.; Hoshino, S.; Yamada, Y.; Shirane, G. NeutronScattering Study on Phase Transitions of $\mathrm{CsPbCl}_{3}$. Phys. Rev. B 1974, 9 (10), 4549-4559.

(19) Worhatch, R. J.; Kim, H.; Swainson, I. P.; Yonkeu, A. L.; Billinge, S. J. L. Study of Local Structure in Selected Organic-Inorganic Perovskites in the $\operatorname{Pm} \overline{3} \mathrm{~m}$ Phase. Chem. Mater. 2008, 20 (4), 12721277.

(20) Bernasconi, A.; Malavasi, L. Direct Evidence of Permanent Octahedra Distortion in $\mathrm{MAPbBr}_{3}$ Hybrid Perovskite. ACS Energy Lett. 2017, 2 (4), 863-868.

(21) Richter, J. M.; Branchi, F.; Valduga de Almeida Camargo, F.; Zhao, B.; Friend, R. H.; Cerullo, G.; Deschler, F. Ultrafast Carrier Thermalization in Lead Iodide Perovskite Probed with Two-Dimensional Electronic Spectroscopy. Nat. Commun. 2017, 8 (1), 376.

(22) Hopper, T. R.; Gorodetsky, A.; Jeong, A.; Krieg, F.; Bodnarchuk, M. I.; Maimaris, M.; Chaplain, M.; Macdonald, T. J.; Huang, X.; Lovrincic, R.; Kovalenko, M. V.; Bakulin, A. A. Hot Carrier Dynamics in Perovskite Nanocrystal Solids: Role of the Cold Carriers, Nanoconfinement, and the Surface. Nano Lett. 2020, 20 (4), 2271-2278.

(23) Boehme, S. C.; Brinck, S. t.; Maes, J.; Yazdani, N.; Zapata, F.; Chen, K.; Wood, V.; Hodgkiss, J. M.; Hens, Z.; Geiregat, P.; Infante, I. Phonon-Mediated and Weakly Size-Dependent Electron and Hole Cooling in $\mathrm{CsPbBr}_{3}$ Nanocrystals Revealed by Atomistic Simulations and Ultrafast Spectroscopy. Nano Lett. 2020, 20, 1819.

(24) Seiler, H.; Palato, S.; Sonnichsen, C.; Baker, H.; Socie, E.; Strandell, D. P.; Kambhampati, P. Two-Dimensional Electronic Spectroscopy Reveals Liquid-like Lineshape Dynamics in $\mathrm{CsPbI}_{3}$ Perovskite Nanocrystals. Nat. Commun. 2019, 10 (1), 4962.

(25) Batignani, G.; Fumero, G.; Kandada, A. R. S.; Cerullo, G.; Gandini, M.; Ferrante, C.; Petrozza, A.; Scopigno, T. Probing Femtosecond Lattice Displacement upon Photo-Carrier Generation in Lead Halide Perovskite. Nat. Commun. 2018, 9 (1), 1-5.

(26) Zhao, W.; Qin, Z.; Zhang, C.; Wang, G.; Dai, X.; Xiao, M. Coherent Exciton-Phonon Coupling in Perovskite Semiconductor Nanocrystals Studied by Two-Dimensional Electronic Spectroscopy. Appl. Phys. Lett. 2019, 115 (24), 243101.

(27) Cinquanta, E.; Meggiolaro, D.; Motti, S. G.; Gandini, M.; Alcocer, M. J. P.; Akkerman, Q. A.; Vozzi, C.; Manna, L.; De Angelis, F.; Petrozza, A.; Stagira, S. Ultrafast THz Probe of Photoinduced Polarons in Lead-Halide Perovskites. Phys. Rev. Lett. 2019, 122 (16), 166601.

(28) Zhu, X.-Y.; Podzorov, V. Charge Carriers in Hybrid OrganicInorganic Lead Halide Perovskites Might Be Protected as Large Polarons. J. Phys. Chem. Lett. 2015, 6 (23), 4758-4761.

(29) Yu, P.; Cardona, M. Fundamentals of Semiconductors: Physics and Materials Properties; Springer Science \& Business Media, 2010.

(30) Wright, A. D.; Verdi, C.; Milot, R. L.; Eperon, G. E.; PérezOsorio, M. A.; Snaith, H. J.; Giustino, F.; Johnston, M. B.; Herz, L. M. Electron-Phonon Coupling in Hybrid Lead Halide Perovskites. Nat. Commun. 2016, 7 (1), 11755.

(31) Ramade, J.; Andriambariarijaona, L. M.; Steinmetz, V.; Goubet, N.; Legrand, L.; Barisien, T.; Bernardot, F.; Testelin, C.; Lhuillier, E.; Bramati, A.; Chamarro, M. Exciton-Phonon Coupling in a CsPbBr Single Nanocrystal. Appl. Phys. Lett. 2018, 112 (7), 072104. 
(32) Zheng, K.; Abdellah, M.; Zhu, Q.; Kong, Q.; Jennings, G.; Kurtz, C. A.; Messing, M. E.; Niu, Y.; Gosztola, D. J.; Al-Marri, M. J.; Zhang, X.; Pullerits, T.; Canton, S. E. Direct Experimental Evidence for Photoinduced Strong-Coupling Polarons in Organolead Halide Perovskite Nanoparticles. J. Phys. Chem. Lett. 2016, 7 (22), 4535-4539.

(33) Liu, C.; Tsai, H.; Nie, W.; Gosztola, D. J.; Zhang, X. Direct Spectroscopic Observation of the Hole Polaron in Lead Halide Perovskites. J. Phys. Chem. Lett. 2020, 11 (15), 6256-6261.

(34) Wu, X.; Tan, L. Z.; Shen, X.; Hu, T.; Miyata, K.; Trinh, M. T.; Li, R.; Coffee, R.; Liu, S.; Egger, D. A.; Makasyuk, I.; Zheng, Q.; Fry, A.; Robinson, J. S.; Smith, M. D.; Guzelturk, B.; Karunadasa, H. I.; Wang, X.; Zhu, X.; Kronik, L.; Rappe, A. M.; Lindenberg, A. M. Light-Induced Picosecond Rotational Disordering of the Inorganic Sublattice in Hybrid Perovskites. Sci. Adv. 2017, 3 (7), No. e1602388.

(35) Guzelturk, B.; Winkler, T.; Van de Goor, T. W. J.; Smith, M. D.; Bourelle, S. A.; Feldmann, S.; Trigo, M.; Teitelbaum, S. W.; Steinrück, H.-G.; de la Pena, G. A.; Alonso-Mori, R.; Zhu, D.; Sato, T.; Karunadasa, H. I.; Toney, M. F.; Deschler, F.; Lindenberg, A. M. Visualization of Dynamic Polaronic Strain Fields in Hybrid Lead Halide Perovskites. Nat. Mater. 2021, 20, 1-6.

(36) Santomauro, F. G.; Grilj, J.; Mewes, L.; Nedelcu, G.; Yakunin, S.; Rossi, T.; Capano, G.; Al Haddad, A.; Budarz, J.; Kinschel, D.; Ferreira, D. S.; Rossi, G.; Gutierrez Tovar, M.; Grolimund, D.; Samson, V.; Nachtegaal, M.; Smolentsev, G.; Kovalenko, M. V.; Chergui, M. Localized Holes and Delocalized Electrons in Photoexcited Inorganic Perovskites: Watching Each Atomic Actor by Picosecond X-Ray Absorption Spectroscopy. Struct. Dyn. 2017, 4 (4), 044002.

(37) Kirschner, M. S.; Diroll, B. T.; Guo, P.; Harvey, S. M.; Helweh, W.; Flanders, N. C.; Brumberg, A.; Watkins, N. E.; Leonard, A. A.; Evans, A. M.; Wasielewski, M. R.; Dichtel, W. R.; Zhang, X.; Chen, L. X.; Schaller, R. D. Photoinduced, Reversible Phase Transitions in AllInorganic Perovskite Nanocrystals. Nat. Commun. 2019, 10 (1), 504.

(38) Puppin, M.; Polishchuk, S.; Colonna, N.; Crepaldi, A.; Dirin, D. N.; Nazarenko, O.; De Gennaro, R.; Gatti, G.; Roth, S.; Barillot, T.; Poletto, L.; Xian, R. P.; Rettig, L.; Wolf, M.; Ernstorfer, R.; Kovalenko, M. V.; Marzari, N.; Grioni, M.; Chergui, M. Evidence of Large Polarons in Photoemission Band Mapping of the Perovskite Semiconductor CsPbBr. Phys. Rev. Lett. 2020, 124 (20), 206402.

(39) Rehr, J. J.; Albers, R. C. Theoretical Approaches to X-Ray Absorption Fine Structure. Rev. Mod. Phys. 2000, 72 (3), 621-654.

(40) Teo, B. K. EXAFS: Basic Principles and Data Analysis; Springer Science \& Business Media, 2012.

(41) Chergui, M.; Collet, E. Photoinduced Structural Dynamics of Molecular Systems Mapped by Time-Resolved X-Ray Methods. Chem. Rev. 2017, 117 (16), 11025-11065.

(42) March, A. M.; Stickrath, A.; Doumy, G.; Kanter, E. P.; Krässig, B.; Southworth, S. H.; Attenkofer, K.; Kurtz, C. A.; Chen, L. X.; Young, L. Development of High-Repetition-Rate Laser Pump/x-Ray Probe Methodologies for Synchrotron Facilities. Rev. Sci. Instrum. 2011, 82 (7), 073110

(43) March, A. M.; Doumy, G.; Andersen, A.; Al Haddad, A.; Kumagai, Y.; Tu, M.-F.; Bang, J.; Bostedt, C.; Uhlig, J.; Nascimento, D. R.; Assefa, T. A.; Németh, Z.; Vankó, G.; Gawelda, W.; Govind, N.; Young, L. Elucidation of the Photoaquation Reaction Mechanism in Ferrous Hexacyanide Using Synchrotron X-Rays with Sub-PulseDuration Sensitivity. J. Chem. Phys. 2019, 151 (14), 144306.

(44) Krieg, F.; Ochsenbein, S. T.; Yakunin, S.; Ten Brinck, S.; Aellen, P.; Süess, A.; Clerc, B.; Guggisberg, D.; Nazarenko, O.; Shynkarenko, Y.; Kumar, S.; Shih, C.-J.; Infante, I.; Kovalenko, M. V. Colloidal $\mathrm{CsPbX}_{3}(\mathrm{X}=\mathrm{Cl}, \mathrm{Br}, \mathrm{I})$ Nanocrystals 2.0: Zwitterionic Capping Ligands for Improved Durability and Stability. ACS Energy Lett. 2018, 3 (3), 641-646.

(45) Giannozzi, P.; Baroni, S.; Bonini, N.; Calandra, M.; Car, R.; Cavazzoni, C.; Ceresoli, D.; Chiarotti, G. L.; Cococcioni, M.; Dabo, I.; Corso, A. D.; Gironcoli, S.; de Fabris, S.; Fratesi, G.; Gebauer, R.; Gerstmann, U.; Gougoussis, C.; Kokalj, A.; Lazzeri, M.; Martin-Samos, L.; Marzari, N.; Mauri, F.; Mazzarello, R.; Paolini, S.; Pasquarello, A.; Paulatto, L.; Sbraccia, C.; Scandolo, S.; Sclauzero, G.; Seitsonen, A. P.; Smogunov, A.; Umari, P.; Wentzcovitch, R. M. QUANTUM
ESPRESSO: A Modular and Open-Source Software Project for Quantum Simulations of Materials. J. Phys.: Condens. Matter 2009, 21 (39), 395502.

(46) Giannozzi, P.; Andreussi, O.; Brumme, T.; Bunau, O.; Nardelli, M. B.; Calandra, M.; Car, R.; Cavazzoni, C.; Ceresoli, D.; Cococcioni, M.; Colonna, N.; Carnimeo, I.; Corso, A. D.; Gironcoli, S.; de Delugas, P.; DiStasio, R. A.; Ferretti, A.; Floris, A.; Fratesi, G.; Fugallo, G.; Gebauer, R.; Gerstmann, U.; Giustino, F.; Gorni, T.; Jia, J.; Kawamura, M.; Ko, H.-Y.; Kokalj, A.; Küçükbenli, E.; Lazzeri, M.; Marsili, M.; Marzari, N.; Mauri, F.; Nguyen, N. L.; Nguyen, H.-V.; Otero-De-LaRoza, A.; Paulatto, L.; Poncé, S.; Rocca, D.; Sabatini, R.; Santra, B.; Schlipf, M.; Seitsonen, A. P.; Smogunov, A.; Timrov, I.; Thonhauser, T.; Umari, P.; Vast, N.; Wu, X.; Baroni, S. Advanced Capabilities for Materials Modelling with Quantum ESPRESSO. J. Phys.: Condens. Matter 2017, 29 (46), 465901.

(47) Perdew, J. P.; Burke, K.; Ernzerhof, M. Generalized Gradient Approximation Made Simple. Phys. Rev. Lett. 1996, 77 (18), 38653868.

(48) Dal Corso, A. Pseudopotentials Periodic Table: From H to Pu. Comput. Mater. Sci. 2014, 95, 337-350.

(49) Taillefumier, M.; Cabaret, D.; Flank, A.-M.; Mauri, F. X-Ray Absorption near-Edge Structure Calculations with the Pseudopotentials: Application to the K Edge in Diamond and $\alpha$-Quartz. Phys. Rev. B: Condens. Matter Mater. Phys. 2002, 66 (19), 195107.

(50) Gougoussis, C.; Calandra, M.; Seitsonen, A. P.; Mauri, F. Firstprinciples calculations of $\mathrm{X}$-ray absorption in a scheme based on ultrasoft pseudopotentials: From $\alpha$-quartz to high- $\mathrm{T}_{\mathrm{c}}$ compounds. Phys. Rev. B: Condens. Matter Mater. Phys. 2009, 80 (7), 075102.

(51) Gilmore, K.; Vinson, J.; Shirley, E. L.; Prendergast, D.; Pemmaraju, C. D.; Kas, J. J.; Vila, F. D.; Rehr, J. J. Efficient Implementation of Core-Excitation Bethe-Salpeter Equation Calculations. Comput. Phys. Commun. 2015, 197, 109-117.

(52) Wang, Y.; Li, X.; Song, J.; Xiao, L.; Zeng, H.; Sun, H. AllInorganic Colloidal Perovskite Quantum Dots: A New Class of Lasing Materials with Favorable Characteristics. Adv. Mater. 2015, 27 (44), 7101-7108.

(53) Diroll, B. T.; Schaller, R. D. Intraband Cooling in All-Inorganic and Hybrid Organic-Inorganic Perovskite Nanocrystals. Adv. Funct. Mater. 2019, 29 (37), 1901725.

(54) Ahmed, T.; Seth, S.; Samanta, A. Boosting the Photoluminescence of $\mathrm{CsPbX}_{3}(\mathrm{X}=\mathrm{Cl}, \mathrm{Br}, \mathrm{I})$ Perovskite Nanocrystals Covering a Wide Wavelength Range by Postsynthetic Treatment with Tetrafluoroborate Salts. Chem. Mater. 2018, 30 (11), 3633-3637.

(55) Hopper, T. R.; Gorodetsky, A.; Frost, J. M.; Müller, C.; Lovrincic, R.; Bakulin, A. A. Ultrafast Intraband Spectroscopy of Hot-Carrier Cooling in Lead-Halide Perovskites. ACS Energy Lett. 2018, 3 (9), 2199-2205

(56) Diroll, B. T.; Schaller, R. D. Heating and Cooling of LigandCoated Colloidal Nanocrystals in Solid Films and Solvent Matrices. Nanoscale 2019, 11 (17), 8204-8209.

(57) Prendergast, D.; Galli, G. X-Ray Absorption Spectra of Water from First Principles Calculations. Phys. Rev. Lett. 2006, 96 (21), 215502 .

(58) Gao, S.-P.; Pickard, C. J.; Payne, M. C.; Zhu, J.; Yuan, J. Theory of Core-Hole Effects in 1s Core-Level Spectroscopy of the First-Row Elements. Phys. Rev. B: Condens. Matter Mater. Phys. 2008, 77 (11), 115122.

(59) Liu, C.; Wang, Y.; Geng, H.; Zhu, T.; Ertekin, E.; Gosztola, D.; Yang, S.; Huang, J.; Yang, B.; Han, K.; Canton, S. E.; Kong, Q.; Zheng, K.; Zhang, X. Asynchronous Photoexcited Electronic and Structural Relaxation in Lead-Free Perovskites. J. Am. Chem. Soc. 2019, 141 (33), 13074-13080.

(60) Penfold, T. J.; Szlachetko, J.; Santomauro, F. G.; Britz, A.; Gawelda, W.; Doumy, G.; March, A. M.; Southworth, S. H.; Rittmann, J.; Abela, R.; Chergui, M.; Milne, C. J. Revealing Hole Trapping in Zinc Oxide Nanoparticles by Time-Resolved X-Ray Spectroscopy. Nat. Commun. 2018, 9 (1), 478.

(61) Koide, A.; Uemura, Y.; Kido, D.; Wakisaka, Y.; Takakusagi, S.; Ohtani, B.; Niwa, Y.; Nozawa, S.; Ichiyanagi, K.; Fukaya, R.; Adachi, S.; 
Katayama, T.; Togashi, T.; Owada, S.; Yabashi, M.; Yamamoto, Y.; Katayama, M.; Hatada, K.; Yokoyama, T.; Asakura, K. Photoinduced Anisotropic Distortion as the Electron Trapping Site of Tungsten Trioxide by Ultrafast $\mathrm{W} \mathrm{L}_{1}$-Edge X-Ray Absorption Spectroscopy with Full Potential Multiple Scattering Calculations. Phys. Chem. Chem. Phys. 2020, 22 (5), 2615-2621.

(62) Ong, W.-L.; Rupich, S. M.; Talapin, D. V.; McGaughey, A. J. H.; Malen, J. A. Surface Chemistry Mediates Thermal Transport in ThreeDimensional Nanocrystal Arrays. Nat. Mater. 2013, 12 (5), 410-415.

(63) Diroll, B. T.; Mannodi-Kanakkithodi, A.; Chan, M. K. Y.; Schaller, R. D. Spectroscopic Comparison of Thermal Transport at Organic-Inorganic and Organic-Hybrid Interfaces Using $\mathrm{CsPbBr}_{3}$ and $\mathrm{FAPbBr}_{3}(\mathrm{FA}=$ Formamidinium $)$ Perovskite Nanocrystals. Nano Lett. 2019, 19 (11), 8155-8160.

(64) Limmer, D. T.; Ginsberg, N. S. Photoinduced Phase Separation in the Lead Halides Is a Polaronic Effect. J. Chem. Phys. 2020, 152 (23), 230901.

(65) Ramade, J.; Andriambariarijaona, L. M.; Steinmetz, V.; Goubet, N.; Legrand, L.; Barisien, T.; Bernardot, F.; Testelin, C.; Lhuillier, E.; Bramati, A.; Chamarro, M. Exciton-Phonon Coupling in a $\mathrm{CsPbr}_{3}$ Single Nanocrystal. Appl. Phys. Lett. 2018, 112 (7), 072104.

(66) Egami, T.; Billinge, S. J. L. Underneath the Bragg Peaks: Structural Analysis of Complex Materials; Newnes, 2012.

(67) Rittmann-Frank, M. H.; Milne, C. J.; Rittmann, J.; Reinhard, M.; Penfold, T. J.; Chergui, M. Mapping of the Photoinduced Electron Traps in $\mathrm{TiO}_{2}$ by Picosecond X-Ray Absorption Spectroscopy. Angew. Chem., Int. Ed. 2014, 53 (23), 5858-5862.

(68) Momma, K.; Izumi, F. VESTA 3 for Three-Dimensional Visualization of Crystal, Volumetric and Morphology Data. J. Appl. Crystallogr. 2011, 44 (6), 1272-1276. 